\title{
Involvement of neutrophil extracellular traps in the pathogenesis of glomerulonephritis in a case of systemic lupus erythematosus and antineutrophil cytoplasmic antibody-associated vasculitis overlap syndrome
}

\author{
Arisa Senda ${ }^{1} \cdot$ Ryutaro Sasai $^{1} \cdot$ Kurumi Kato $^{2} \cdot$ Yuka Nishibata $^{2} \cdot$ Sakiko Masuda $^{2} \cdot$ Akihiro Ishizu $^{2} \cdot$ Noriko Takahara $^{1}$
}

Received: 5 August 2021 / Accepted: 23 December 2021 / Published online: 13 January 2022

(c) The Author(s) 2022

\begin{abstract}
Systemic lupus erythematosus (SLE) and antineutrophil cytoplasmic antibody-associated vasculitis (AAV) are autoimmune diseases that often cause rapidly progressive glomerulonephritis, with neutrophil extracellular traps (NETs) involved in their pathogenesis. However, the involvement of NETs in the renal damage caused by SLE/AAV overlap syndrome has not been clarified yet. In this study, we detected renal deposition of NETs in a patient with SLE/AAV overlap syndrome. In addition, a significantly increased level of NET-inducing activity was observed in the patient's serum, which improved with treatment. On the other hand, a markedly lower level of NET degradation was observed in the patient's serum as compared to healthy subjects' sera, without any posttreatment changes. These findings suggest that NETs may play a role in the pathogenesis of renal injury associated with SLE/AAV overlap syndrome.
\end{abstract}

Keywords Systemic lupus erythematosus $\cdot$ Neutrophil extracellular traps $\cdot$ ANCA-associated vasculitis

\section{Introduction}

Systemic lupus erythematosus (SLE) and antineutrophil cytoplasmic antibody (ANCA)-associated vasculitis (AAV) are autoimmune diseases that often cause rapidly progressive glomerulonephritis (RPGN). In SLE, excessively produced autoantibodies-especially anti-deoxyribonucleic acid (DNA) antibodies-bind to their respective antigens (e.g., DNA fibers) to form immune complexes, which are deposited in the kidneys and other organs, and subsequently induce inflammation through activation of the complement system [1].

On the other hand, AAV is characterized by inflammation of small blood vessels, mainly affecting the kidneys and lungs [2]. In AAV, myeloperoxidase (MPO) and proteinase

Noriko Takahara

norikot@amh.ako.hyogo.jp

Department of Internal Medicine, Ako Municipal Hospital, 1090 Nakahiro, Ako, Hyogo 678-0232, Japan

2 Department of Medical Laboratory Science, Faculty of Health Sciences, Hokkaido University, Kita-12, Nishi-5, Kita-ku, Sapporo, Hokkaido 060-0812, Japan
3 (PR3), which are proteins mainly expressed in neutrophil granules, develop antigenicity, leading to ANCA production. Neutrophils activated by ANCA binding can then infiltrate into the local glomerular area, causing necrotizing crescentic glomerulonephritis.

Recently, it has been suggested that neutrophil extracellular traps (NETs) are associated with a variety of autoimmune diseases, such as SLE and AAV. NETs are extracellular DNA structures that are involved in a powerful immune mechanism whereby neutrophils capture and kill microorganisms by releasing bactericidal proteins and DNA fibers into the extracellular space [3]. It has been suggested that dysregulation in NET formation has an important role in the production of anti-DNA antibodies and myeloperoxidaseantineutrophil cytoplasmic antibody (MPO-ANCA) [4, 5]. Furthermore, it has been shown that sera from SLE and AAV patients induce NETs in healthy neutrophils, but the degradation rate of NETs is significantly lower than that of healthy sera [6]. Thus, evaluating serum NET induction and degradation activities is helpful in understanding the NETmediated pathology underlying SLE and AAV.

Here, we report the case of RPGN in a patient with SLE/ AAV overlap syndrome. The involvement of NETs in the 
renal damage caused by SLE/AAV overlap syndrome has not been reported previously. Further, we investigated (1) NET deposition in the patient's renal tissue and (2) NET-inducing and NET-degrading activities in the patient's serum as well as their posttreatment changes.

\section{Case report}

A 36-year-old woman presented to our hospital with a fever of $39{ }^{\circ} \mathrm{C}$ for a month, dry cough, redness in both eyes, polyarthralgia, purpura, subcutaneous bleeding, edema of the lower legs, nonscarring alopecia, and stomatitis. Laboratory findings were remarkable for proteinuria, hematuria, elevated serum creatinine concentration (estimated glomerular filtration rate of $59.0 \mathrm{~mL} / \mathrm{min} / 1.73 \mathrm{~m}^{2}$ ), MPOANCA $>300 \mathrm{IU} / \mathrm{mL}$, low complement levels, and positive anti-double-stranded DNA (anti-dsDNA) antibody (Table 1). A biopsy specimen was obtained from the rash on the lower leg. Perivascular infiltration of inflammatory cells, including neutrophils, with nuclear dust around the vessels in the upper dermis, which was consistent with leukocytoclastic vasculitis, was noted (Fig. 1).

Renal biopsy revealed that a total of 44 glomeruli, including 8 with cellular crescent, 3 with fibrinoid necrosis, and 2 with endocapillary hypercellularity but no global sclerosis, were observed. Focal interstitial inflammation with edema was also observed, although there was no overt peritubular
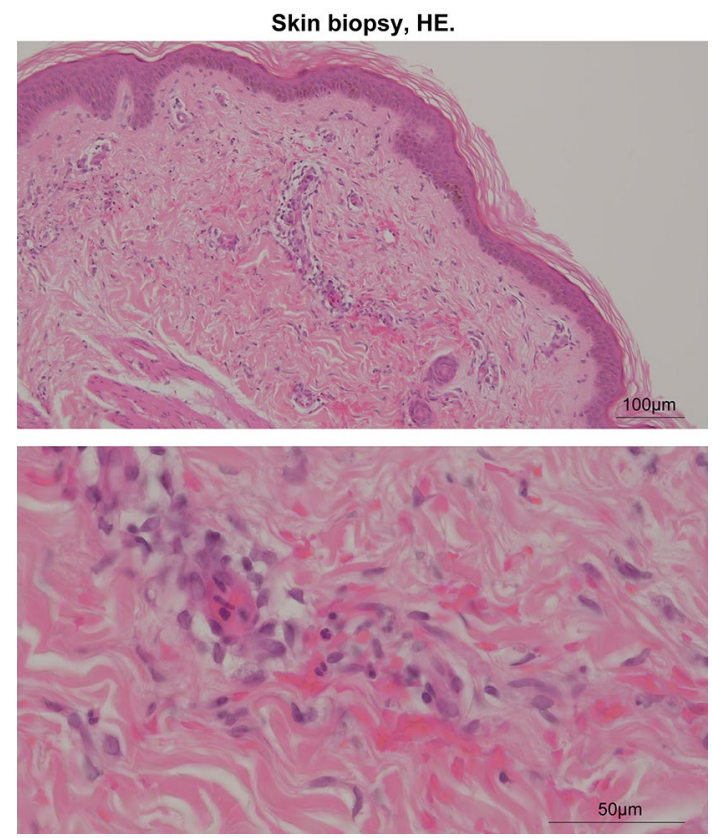

Fig. 1 Skin biopsy findings (hematoxylin and eosin). A basket-weave pattern of the epidermis was observed. Infiltration of inflammatory cells, including neutrophils, with nuclear dust around the vessels in the upper dermis and extravasation of red blood cells were observed

capillaritis (Fig. 2a). Immunofluorescence staining showed granular depositions of $\operatorname{IgG}( \pm), \operatorname{IgA}(1+), \mathrm{C} 3(1+), \mathrm{C} 1 \mathrm{q}(1+)$ and $\operatorname{IgM}(1+)$ along the capillary wall and partly in the
Table 1 Laboratory findings on admission

\begin{tabular}{|c|c|c|c|c|c|}
\hline \multicolumn{2}{|c|}{ Peripheral blood } & \multicolumn{2}{|c|}{ Blood chemistry } & \multicolumn{2}{|l|}{ Serology } \\
\hline $\mathrm{RBC}$ & $330 \times 10^{4} / \mu \mathrm{L}$ & CRP & $2.46 \mathrm{mg} / \mathrm{dL}$ & ANA & 640 times \\
\hline $\mathrm{Hb}$ & $10.3 \mathrm{~g} / \mathrm{dL}$ & $\mathrm{TP}$ & $7.7 \mathrm{~g} / \mathrm{dL}$ & Anti-dsDNA antibody & $125 \mathrm{IU} / \mathrm{mL}$ \\
\hline $\mathrm{Ht}$ & $30.3 \%$ & CK & 74 U/L & $\mathrm{RF}$ & $7 \mathrm{IU} / \mathrm{mL}$ \\
\hline Plt & $36.0 \times 10^{4} / \mu \mathrm{L}$ & T-Bil & $0.5 \mathrm{mg} / \mathrm{dL}$ & aCL-IgG & $13 \mathrm{U} / \mathrm{mL}$ \\
\hline WBC & $4200 / \mu \mathrm{L}$ & AST & $56 \mathrm{U} / \mathrm{L}$ & $\mathrm{C} 3$ & $46 \mathrm{mg} / \mathrm{dL}$ \\
\hline $\mathrm{Neu}$ & $79.5 \%$ & ALT & $31 \mathrm{U} / \mathrm{L}$ & $\mathrm{C} 4$ & $7 \mathrm{mg} / \mathrm{dL}$ \\
\hline Mono & $1.1 \%$ & LDH & $314 \mathrm{U} / \mathrm{L}$ & $\mathrm{CH} 50$ & $15.5 \mathrm{U} / \mathrm{L}$ \\
\hline Lympho & $15.2 \%$ & Uric acid & $4.8 \mathrm{mg} / \mathrm{dL}$ & MPO-ANCA & $>300.0 \mathrm{IU} / \mathrm{mL}$ \\
\hline Eosino & $1.0 \%$ & BUN & $14 \mathrm{mg} / \mathrm{dL}$ & PR3-ANCA & $1 \mathrm{IU} / \mathrm{mL}$ \\
\hline \multirow[t]{5}{*}{ Baso } & $2.0 \%$ & $\mathrm{Cr}$ & $0.88 \mathrm{mg} / \mathrm{dL}$ & & \\
\hline & & $\mathrm{Na}$ & $133 \mathrm{mEq} / \mathrm{L}$ & Urinalysis & \\
\hline & & $\mathrm{K}$ & $4.5 \mathrm{mEq} / \mathrm{L}$ & $\mathrm{RBC}$ & 300-599/HPF \\
\hline & & $\mathrm{Cl}$ & $99 \mathrm{mEq} / \mathrm{L}$ & WBC & $5-9 / \mathrm{HPF}$ \\
\hline & & $\mathrm{Ca}$ & $8.6 \mathrm{mg} / \mathrm{dL}$ & Protein/Cr & $1.09 \mathrm{~g} / \mathrm{g}-\mathrm{Cr}$ \\
\hline
\end{tabular}

$R B C$ red blood cell, $H b$ hemoglobin, $H t$ hematocrit, Plt platelet, $W B C$ white blood cell, Neu neutrophil, Mono monocyte, Lympho lymphocyte, Eosino eosinophil, Baso basophil, CRP C-reactive protein, TP total protein, $C K$ creatinine kinase, $T$-Bil total bilirubin, $A S T$ aspartate aminotransferase, $A L T$ alanine aminotransferase, $L D H$ lactate dehydrogenase, $B U N$ blood urea nitrogen, $\mathrm{Cr}$ creatinine, $\mathrm{Na}$ sodium, $\mathrm{K}$ potassium, $\mathrm{Cl}$ chloride, $\mathrm{Ca}$ calcium, $A N A$ anti-nuclear antibody, Anti-dsDNA anti-double stranded deoxyribonucleic acid, $R F$ rheumatoid factor, $a C L-I g G$ anti-cardiolipin immunoglobulin $\mathrm{G}, C 3$ complement $3, C 4$ complement4, CH50 50\% hemolytic complement activity, MPO-ANCA myeloperoxidase-anti-neutrophil cytoplasmic antibody, $P R$-ANCA proteinase-3-anti-neutrophil cytoplasmic antibody, $H P F$ high power field 
(a) Renal biopsy, HE.

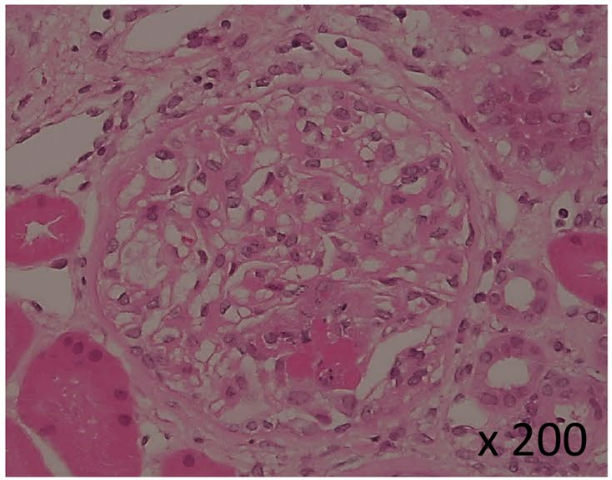

(b) Renal biopsy, IF.
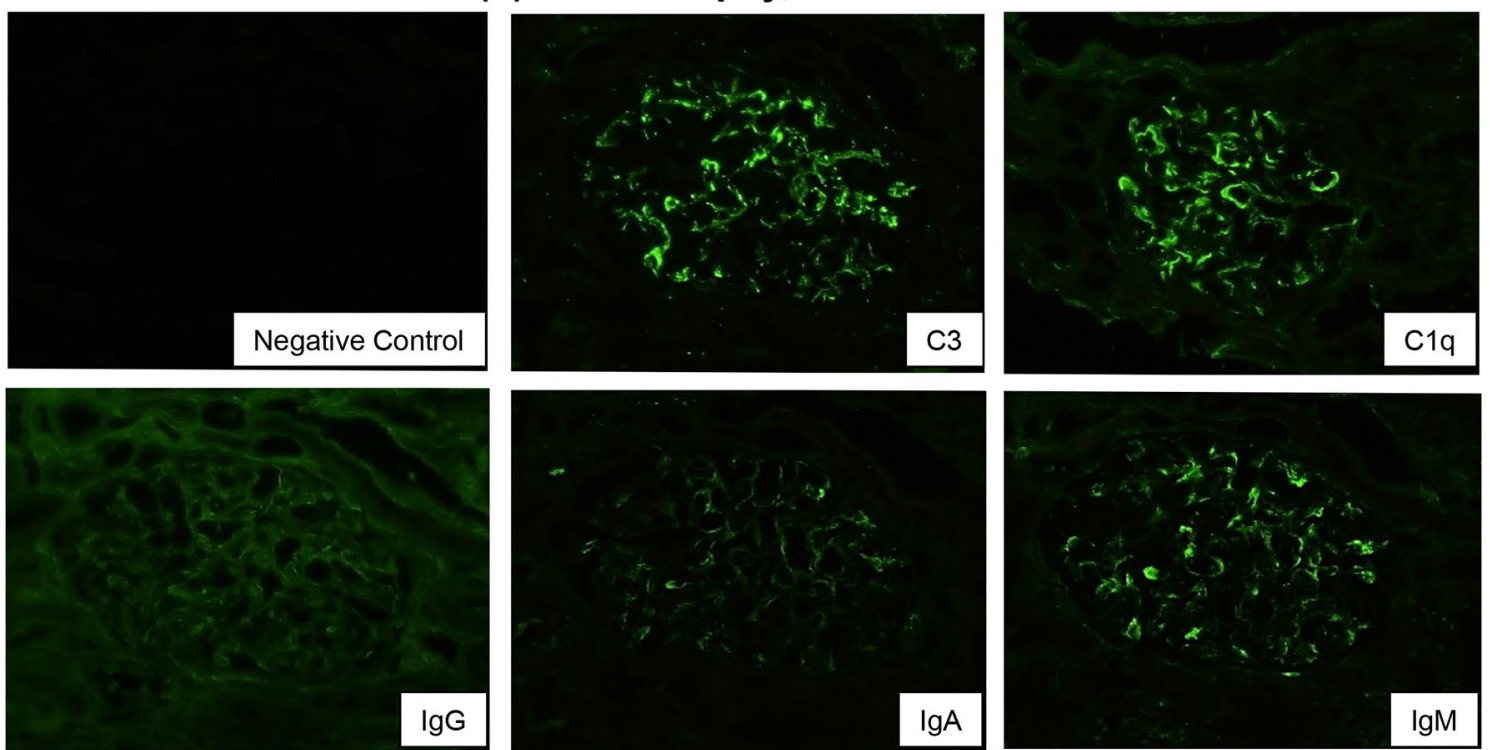

(c) Renal biopsy, EM.

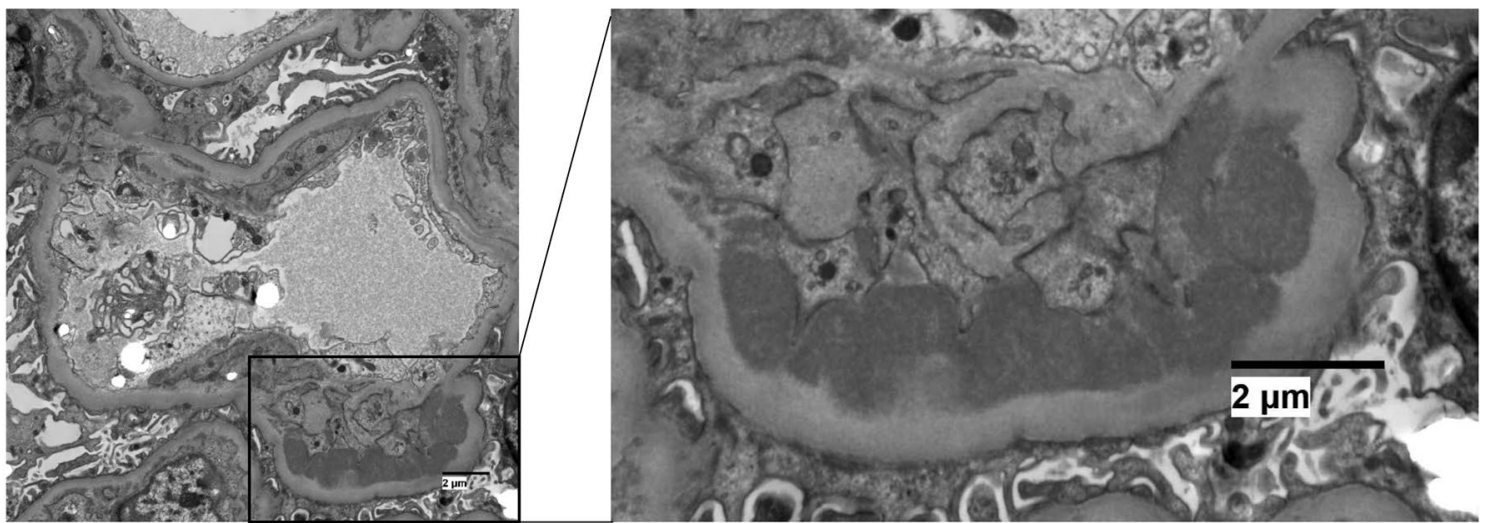

Fig. 2 Renal biopsy findings. a Fibrinoid necrosis, hematoxylin and eosin staining, b immunofluorescence staining, and $\mathbf{c}$ electron-dense deposits in the subendothelium, electron microscopy 
mesangial region (Fig. 2b). Electron microscopy showed dense deposits in the subendothelial and mesangial regions, but no fingerprinting (Fig. 2c). Based on these findings, the patient met the 2019 European League Against Rheumatism (EULAR)/American College of Rheumatology (ACR) classification criteria for SLE diagnosis and was diagnosed as having SLE with type III lupus nephritis (LN). In addition, the patient had a high serum MPO-ANCA level and leukocytoclastic vasculitis in the skin; hence, SLE/AAV overlap syndrome was suspected.

After renal biopsy, on post-admission day (PAD) 3, oral administration of prednisolone (PSL) $1 \mathrm{mg} / \mathrm{kg}$ /day was started. The patient's symptoms such as fever and skin rash improved upon treatment with PSL, but her renal function deteriorated further. On PAD 13, $1 \mathrm{~g}$ /day of mycophenolate mofetil (MMF) was added to the therapeutic regimen, followed by gradual dose increments. On PAD 25, methylprednisolone was also administered at $1 \mathrm{~g} /$ day for 3 days, whereupon her renal function gradually improved. On PAD $41,200 \mathrm{mg}$ /day of hydroxychloroquine (HCQ) was added. With this series of treatments, clinical scores such as SLE Disease Activity Index (SLEDAI) and Birmingham Vasculitis Activity Score (BVAS) improved and anti-dsDNA antibody and MPO-ANCA levels significantly decreased, but the decrease in MPO-ANCA levels was slower than the decrease in anti-dsDNA antibody levels (Fig. 3). On PAD 40, anti-dsDNA antibody and C3 levels normalized, but MPO-ANCA levels, serum creatinine levels, and proteinuria did not improve sufficiently. MPO-ANCA remained weakly positive at $3.6 \mathrm{IU} / \mathrm{mL}$, even after 6 months. Based on this discrepancy between anti-dsDNA antibodies and MPOANCA in response to the therapy, we considered that AAV overlapped with SLE in this patient.

\section{NET deposition in renal tissue}

To clarify the involvement of NETs in the pathogenesis of renal damage, we investigated the deposition of NETs in the patient's renal tissue. Immunofluorescence analyses were performed as previously reported [7]. In brief, citrullinated histone $\mathrm{H} 3$ (Cit-H3) was stained using anti-Cit-H3 antibody (Abcam, ab5103); DNA, using 4'6-diamidino2-phenylindole (DAPI; Sigma-Aldrich, St. Louis, MO); and MPO, using anti-MPO antibody (R\&D Systems, AF3776) to visualize NETs. Merged images of the stained, formalinfixed, paraffin-embedded sections of the patient's renal tissue showed colocalization of MPO and $\mathrm{Cit}-\mathrm{H} 3$ within fibrinoid necrosis in the glomeruli, confirming the deposition of NETs (Fig. 4). The deposition of NETs was also observed in the tubular interstitial space focally.

\section{NET-inducing and NET-degrading activities}

Serological analyses were approved by the Ethical Committee of Ako Municipal Hospital (Permission No. 2020-0004). After obtaining written informed consent from the patient,
Fig. 3 Clinical course. Serum samples were collected at the following time points: (1) on admission, (2) 8 days after starting treatment with PSL, (3) 10 days after initiating MMF administration, (4) 14 days after starting mPSL administration, (5) 10 days after initiating HCQ therapy ( $m P S L$ methylprednisolone, $P S L$ prednisolone, $M M F$ mycophenolate mofetil, $H C Q$ hydroxychloroquine). $\mathrm{Cr}$ creatinine, $u-R B C$ urinary red blood cells, $u$-protein urinary protein, C3 complement 3, SLEDAI Systemic Lupus Erythematosus Disease Activity Index, BVAS Birmingham Vasculitis Activity Score.

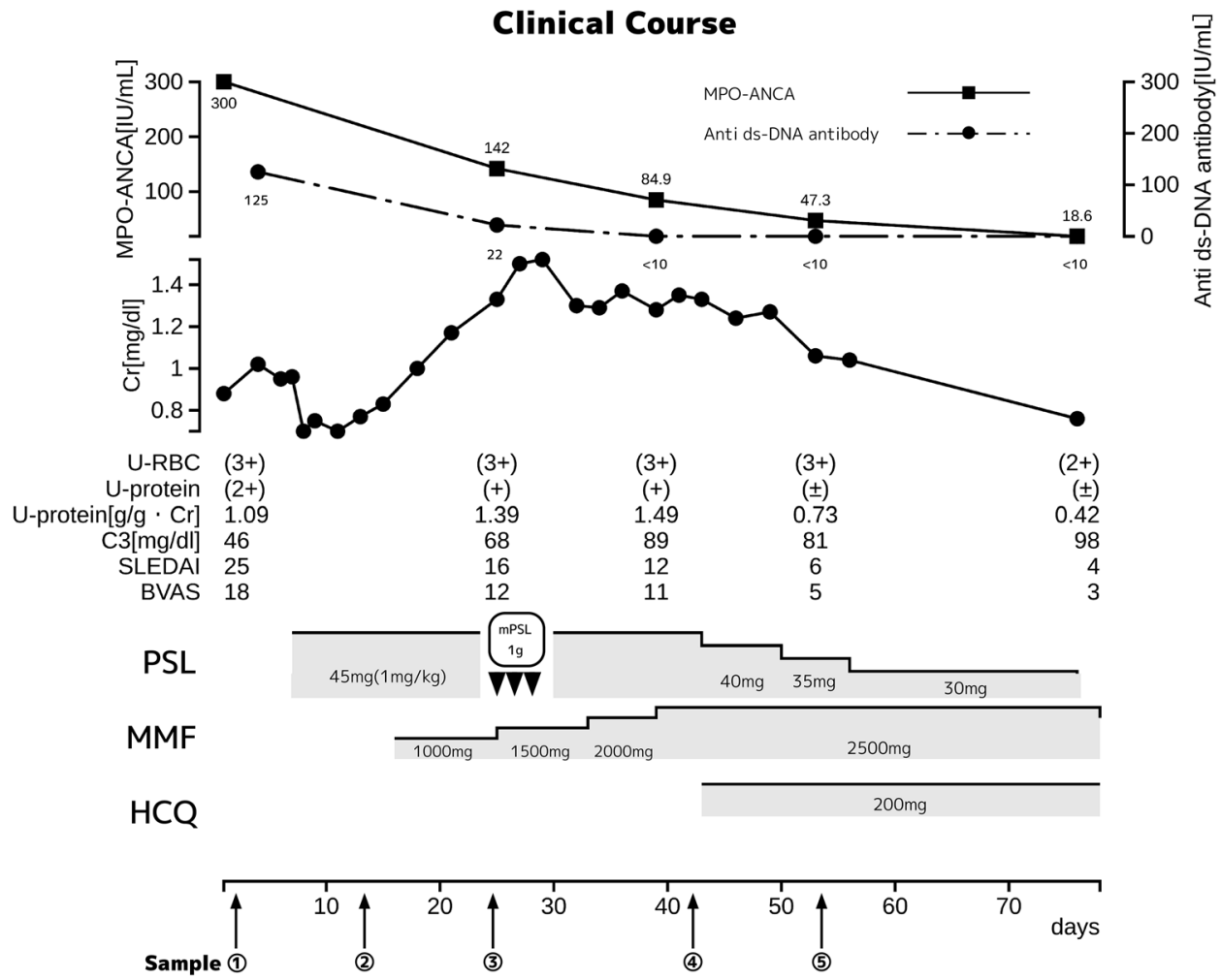


Fig. 4 Immunofluorescent findings in the kidney. Immunofluorescent findings showing the colocalization of myeloperoxidase (MPO) and citrullinated histone $\mathrm{H} 3$ (Cit $\mathrm{H} 3$ ) within fibrinoid necrosis in the glomeruli, confirming the deposition of neutrophil extracellular traps. Original magnification, $\times 400$

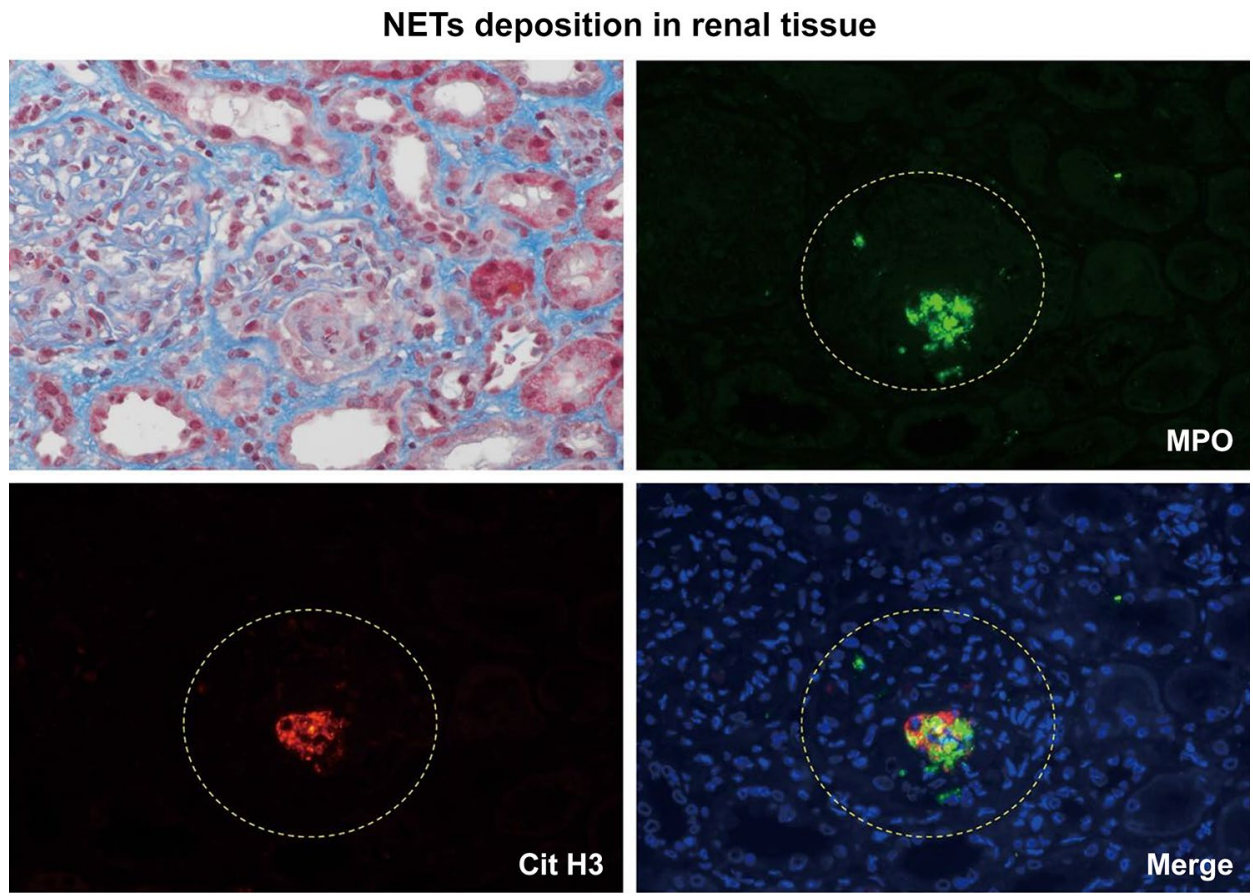

we collected serum samples at the following time points: (1) on admission, (2) 8 days after starting treatment with PSL $45 \mathrm{mg} /$ day ( $1 \mathrm{mg} / \mathrm{kg} /$ day), (3)10 days after initiating MMF administration, (4) 14 days after starting methylprednisolone administration, and (5) 10 days after initiating HCQ therapy (Fig. 3).

First, we examined whether the patient's sera could induce NETs, as described previously [6]. Neutrophils were extracted from the peripheral blood samples of healthy volunteers. The neutrophils were seeded on chamber slides $\left(1 \times 10^{6} / \mathrm{mL}\right)$, incubated at $37^{\circ} \mathrm{C}$ for $30 \mathrm{~min}$, pretreated with or without $5 \mathrm{ng} / \mathrm{mL}$ TNF- $\alpha$ for $15 \mathrm{~min}$, and then incubated with $10 \%$ patient's serum. After incubation at $37^{\circ} \mathrm{C}$ for $4 \mathrm{~h}$, the supernatant was removed, and the remaining cells on the slide were fixed with $4 \%$ paraformaldehyde and sealed with DAPI-containing mounting agent. Photographs of random fields were obtained using a fluorescence microscope (magnification $200 \times$ or $400 \times$ ), NET formation in each sample was assessed as DAPI-positive extracellular chromatin fibers using ImageJ software (NIH, Bethesda, MD, USA), and NET area/neutrophil count was quantified.

Patient's sera collected at untreated time points (1) showed high NET-inducing activity against unstimulated neutrophils from healthy subjects (Fig. 5a); this activity was markedly reduced when PSL was administered at $45 \mathrm{mg} /$ day. With further intensification of the treatment (2)-(5), the NET-inducing activity decreased to the same level as in healthy subjects. Moreover, sera from untreated patients also showed significantly higher NET-inducing activity against neutrophils from healthy subjects primed with TNF- $\alpha$
(Fig. 5b). In contrast to the results for unstimulated neutrophils, the treatment-induced improvement in NET-inducing activity for neutrophils primed with $\mathrm{TNF}-\alpha$ was less evident.

As previously reported, we also examined the NETdegrading activity in the serum of this patient [6]. Peripheral blood neutrophils from healthy volunteers were seeded into slide chambers $\left(1 \times 10^{6} / \mathrm{mL}\right)$, incubated at $37^{\circ} \mathrm{C}$ for $30 \mathrm{~min}$, and then reacted with $100 \mathrm{nM}$ phorbol myristate acetate (Sigma-Aldrich, St. Louis, MO) for $3 \mathrm{~h}$ at $37{ }^{\circ} \mathrm{C}$. The cells were washed with phosphate-buffered saline (PBS), following which $10 \%$ serum sample (1)-(5) was added and incubated at $37{ }^{\circ} \mathrm{C}$ for $6 \mathrm{~h}$. After washing again with PBS, the cells remaining on the slide were fixed with $4 \%$ paraformaldehyde and mounted with a DAPI-containing solution. Photographs of random fields were obtained using a fluorescence microscope (magnification 200x), and the residual NETs area was determined using ImageJ software (6 fields/ chamber slide). NETs degradation rate (\%) was calculated as follows: [(residual NETs incubated with PBS - residual NETs incubated with serum)/residual NETs incubated with PBS $\times 100$. The NET-degrading activity of the patient's serum before treatment initiation was found to be lower than that of the healthy controls' sera (Fig. 6). Furthermore, there was no change in the NET-degrading activity with treatment. 
(a)

NETs induction activity in patient's serum

(for unstimulated healthy neutrophils)

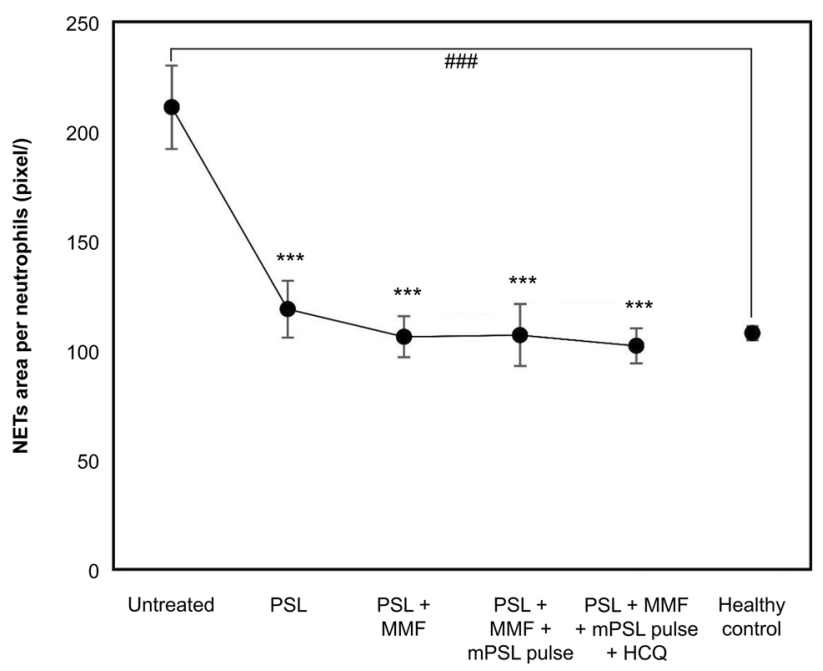

Fig. 5 Neutrophil extracellular traps (NETs) induction activity in patient's serum. The activities were examined against a unstimulated and $\mathbf{b}$ primed neutrophils. Data are shown as the mean \pm SD of four to six samples. $* p<0.05, * * p<0.01, * * * p<0.001$ vs. untreated,

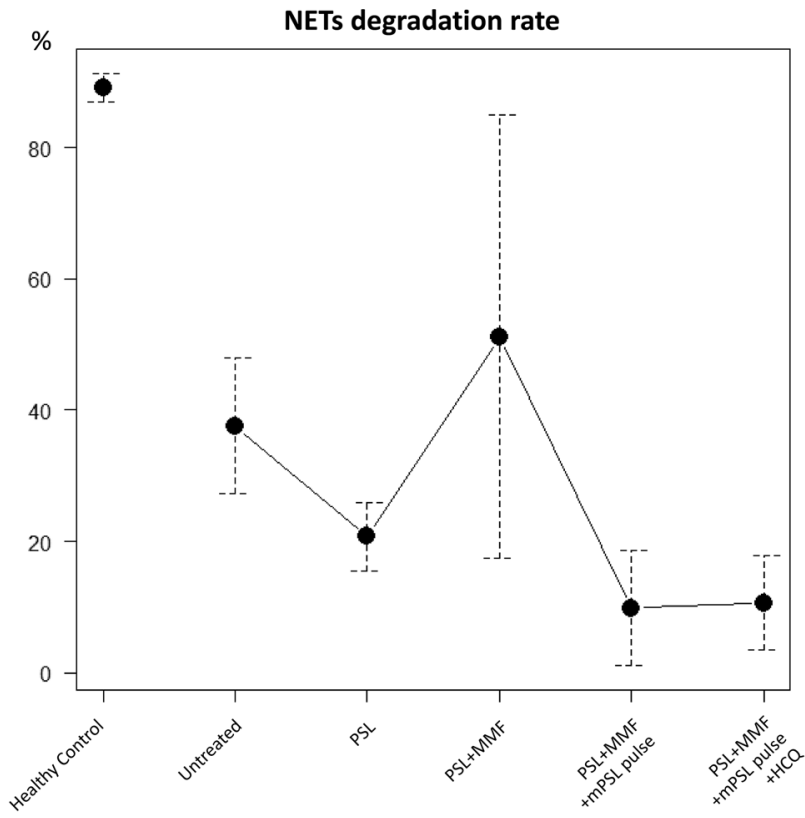

Fig. 6 Neutrophil extracellular traps (NETs) degradation rate. Data are shown as the mean \pm SD of five to seven samples

\section{Discussion}

The case presented herein was diagnosed as an SLE/AAV overlap syndrome causing RPGN, with NET deposition observed at the site of fibrinoid necrosis in the glomeruli. (b) NETs induction activity in patient's serum (for primed neutrophils)

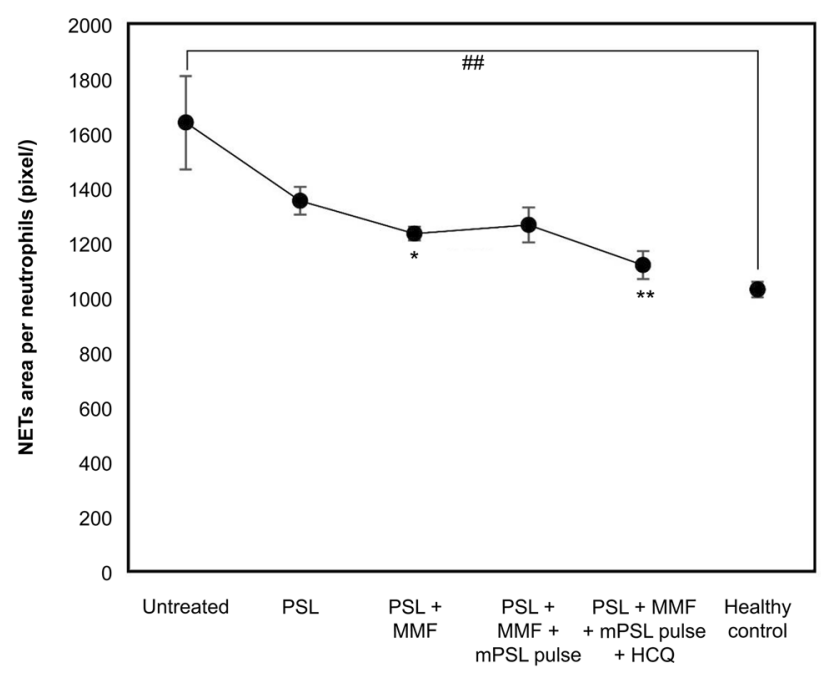

${ }^{\# \#} p<0.01,{ }^{\# \#} p<0.001$ in pairwise comparison using $t$ tests (Bonferroni correction). NET neutrophil extracellular trap, PSL prednisolone, $M M F$ mycophenolate mofetil, $m P S L$ methylprednisolone, $H C Q$ hydroxychloroquine

Besides, the NET-inducing activity in the patient's serum, which was significantly high, improved with treatment.

The patient was diagnosed with SLE based on the 2019 EULAR/ACR classification criteria for SLE. A renal biopsy revealed type III LN with multiple immune complex deposits. In addition, the patient had very high serum MPO-ANCA levels (> $300 \mathrm{IU} / \mathrm{mL}$ ) and leukocytoclastic vasculitis in the skin, leading to the suspicion of an overlap with AAV. The concept of SLE/AAV overlap syndrome is a rare condition that was proposed in 2008 [8]. Although this case met the criteria for SLE, the diagnosis of AAV was based solely on our findings (i.e., elevated MPO-ANCA level, purpura, and subcutaneous bleeding), which did not fully meet the AAV definitions presented by the Japan Research Committee of the Ministry of Health, Labor, and Welfare. However, according to previous reports, patients with SLE/AAV overlap syndrome do not always meet the diagnostic criteria for both, and one or the other feature may be more prominent [9]. In some cases, the symptoms of both diseases may present at different times. On the other hand, it has already been reported that $16-42 \%$ of SLE cases and $37-53 \%$ of LN cases are ANCA positive [10]. LN patients with ANCA positivity tend to have a higher clinical severity than those without ANCA. These findings suggest that in patients with SLE/ AAV overlap syndrome, these two diseases may not only coexist but also interact to exacerbate the condition.

Although the involvement of NETs in the pathogenesis of LN and AAV-related nephritis has been reported recently [11], the significance of NETs in renal damage secondary to SLE/AAV overlap syndrome remains to be elucidated. 
Scattered images taken after immunofluorescence staining of the patient's renal biopsy sections demonstrated colocalization of MPO and Cit-H3 within impaired glomeruli, confirming deposition of NETs.

NET formation (NETosis) by neutrophils is a crucial mechanism for innate immunity [3]. Neutrophils attack pathogens either directly or by releasing NETs, which contain nuclear materials such as DNA and MPO, into the extracellular space. Overproduction of NETs and the persistence of undegraded NETs may further promote the production of autoantibodies against dsDNA fibers and MPO, leading to a vicious cycle and a self-perpetuating condition. NETs are not only immunogenic, but also cytotoxic by nature. Murine studies have shown that NET-derived histones can cause crescentic glomerulonephritis [12]. It has been demonstrated that extracellular MPO, a component of NETs, is involved in oxidative stress production in AAV-related nephritis [13]. NETs present in the kidney are known to be associated with the degree of proteinuria and to drive glomerular endothelial-to-mesenchymal transition in patients with SLE [14]. Here, the deposition of NETs coincided with the site of glomerular necrosis or destruction, suggesting that NETs might have been involved in the development of RPGN in our patient.

Furthermore, we demonstrated that the NET-inducing activity in the patient's serum was high before therapy initiation and later improved with the treatment. It has been reported that NET-inducing activities in serum are increased in both patients with SLE [15] and AAV [16]. Interestingly, however, various characteristic differences in the ex vivo formation of NETs (including morphology, release kinetics, triggers, and pathways) have been shown to exist between SLE and AAV [17]. Therefore, we examined the NET-inducing activity of SLE/AAV overlap syndrome in patient's serum in two ways, using both unstimulated and TNF- $\alpha$-stimulated neutrophils from healthy subjects. MPO appears on the cell surface when neutrophils are stimulated with TNF- $\alpha$, so using TNF- $\alpha$-stimulated neutrophils may reflect NET-inducing activity through ANCAs [6]. In the case of unstimulated neutrophils, NET induction is thought to have been triggered by immune complexes and damageassociated molecular patterns in the patient's serum; this activity was rapidly ameliorated by $1 \mathrm{mg} / \mathrm{kg} /$ day of PSL. However, the therapeutic effect of PSL alone on the NETinducing activity of the patient's serum against neutrophils stimulated with TNF- $\alpha$ turned out to be insufficient, requiring further immunosuppressive agents and higher doses of PSL. In the present case, the decrease in the NET-inducing activity against unstimulated neutrophils was consistent with the improvement in the clinical markers of SLE, such as anti-dsDNA antibody levels and serum complement titers. In contrast, the decrease in the serum NET-inducing activity against TNF- $\alpha$-stimulated neutrophils was consistent with the changes in MPO-ANCA levels. Furthermore, ANCA with NET-inducing activity was found in the serum even when anti-ds-DNA antibody and serum $\mathrm{C} 3$ were negative. These findings suggest that the NET-inducing activity on unstimulated and TNF- $\alpha$-stimulated neutrophils reflects the disease activity of SLE and AAV, respectively. Furthermore, we considered that the AAV lesions in this patient may have had a slightly lower initial response to this treatment than the coexisting SLE lesion.

Moreover, the NET-degrading activity in the patient's serum was low and exhibited no response to the treatment. This was consistent with a previous study demonstrating that NET-degrading activity in the sera of patients with SLE and $\mathrm{AAV}$ was low regardless of their disease activity, thereby suggesting it to be the cause rather than the result of the diseases [6].

In conclusion, NETs may be involved in the pathogenesis of renal injury in SLE/AAV overlap syndrome. Standard treatment for LN significantly improved various clinical symptoms, ameliorated renal dysfunction, and decreased autoantibody titers and NET induction activity in the serum. The patients' clinical course suggests that the NET-inducing activity in the patients' serum was related to two different pathologies-SLE and AAV.

\section{Declarations}

Conflict of interest The authors have declared that no conflict of interest exists.

Ethical approval All procedures performed in studies involving human participants were in accordance with the ethical standards of the institutional research committee at which the studies were conducted (IRB approval number 2020-0004) and with the 1964 Helsinki Declaration and its later amendments or comparable ethical standards.

Informed consent Informed consent was obtained from all individual participants included in the study.

Open Access This article is licensed under a Creative Commons Attribution 4.0 International License, which permits use, sharing, adaptation, distribution and reproduction in any medium or format, as long as you give appropriate credit to the original author(s) and the source, provide a link to the Creative Commons licence, and indicate if changes were made. The images or other third party material in this article are included in the article's Creative Commons licence, unless indicated otherwise in a credit line to the material. If material is not included in the article's Creative Commons licence and your intended use is not permitted by statutory regulation or exceeds the permitted use, you will need to obtain permission directly from the copyright holder. To view a copy of this licence, visit http://creativecommons.org/licenses/by/4.0/. 


\section{References}

1. Davidson A. What is damaging the kidney in lupus nephritis? Nat Rev Rheumatol. 2016;12:143-53.

2. Berden AE, Ferrario F, Hagen EC, et al. Histopathologic classification of ANCA-associated glomerulonephritis. J Am Soc Nephrol. 2010;21:1628-36.

3. Brinkmann V, Reichard U, Goosmann C, et al. Neutrophil extracellular traps kill bacteria. Science. 2004;303:1532-5.

4. Hakkim A, Fürnrohr BG, Amann K, et al. Impairment of neutrophil extracellular trap degradation is associated with lupus nephritis. Proc Natl Acad Sci USA. 2010;107:9813-8.

5. Nakazawa D, Tomaru U, Suzuki A, et al. Abnormal conformation and impaired degradation of neutrophil extracellular traps induced by propylthiouracil: implication of disordered neutrophil extracellular traps in a rat model of myeloperoxidase antineutrophil cytoplasmic antibody-associated vasculitis. Arthritis Rheumatol. 2012;64:3779-87.

6. Nakazawa D, Shida H, Tomaru U, et al. Enhanced formation and disordered regulation of NETs in myeloperoxidase-ANCA-associated microscopic polyangiitis. J Am Soc Nephrol. 2014;25:990-7.

7. Hasegawa J, Wakai S, Kono M, et al. An autopsy case of myeloperoxidase-anti-neutrophil cytoplasmic antibody (MPO-ANCA)associated vasculitis accompanied by cryoglobulinemic vasculitis affecting the kidneys, skin, and gastrointestinal tract. Intern Med. 2018;57:2739-45.

8. Nasr SH, D'Agati VD, Park HR, et al. Necrotizing and crescentic lupus nephritis with antineutrophil cytoplasmic antibody seropositivity. Clin J Am Soc Nephrol. 2008;3:682-90.

9. Jarrot PA, Chiche L, Hervier B, et al. Systemic lupus erythematosus and antineutrophil cytoplasmic antibody-associated vasculitis overlap syndrome in patients with biopsy-proven glomerulonephritis. Medicine (Baltimore). 2016;95: e3748.
10. Wang S, Shang J, Xiao J, et al. Clinicopathologic characteristics and outcomes of lupus nephritis with positive antineutrophil cytoplasmic antibody. Ren Fail. 2020;42:244-54.

11. van Dam LS, Rabelink TJ, van Kooten C, et al. Clinical implications of excessive neutrophil extracellular trap formation in renal autoimmune diseases. Kidney Int Rep. 2018;4:196-211.

12. Kumar SVR, Kulkarni OP, Mulay SR, et al. Neutrophil extracellular trap-related extracellular histones cause vascular necrosis in severe GN. J Am Soc Nephrol. 2015;26:2399-413.

13. Saffarzadeh M, Juenemann C, Queisser MA, et al. Neutrophil extracellular traps directly induce epithelial and endothelial cell death: a predominant role of histones. PLoS One. 2012;7: e32366.

14. Pieterse E, Rother N, Garsen M, et al. Neutrophil extracellular traps drive endothelial-to-mesenchymal transition. Arterioscler Thromb Vasc Biol. 2017;37:1371-9.

15. Kraaij T, Kamerling SWA, de Rooij ENM, et al. The NET-effect of combining rituximab with belimumab in severe systemic lupus erythematosus. J Autoimmun. 2018;91:45-54.

16. Kraaij T, Kamerling SWA, van Dam LS, et al. Excessive neutrophil extracellular trap formation in ANCA-associated vasculitis is independent of ANCA. Kidney Int. 2018;94:139-49.

17. van Dam LS, Kraaij T, Kamerling SWA, et al. Intrinsically distinct role of neutrophil extracellular trap formation in antineutrophil cytoplasmic antibody-associated vasculitis compared to systemic lupus erythematosus. Arthritis Rheumatol. 2019;71:2047-58.

Publisher's Note Springer Nature remains neutral with regard to jurisdictional claims in published maps and institutional affiliations. 